tomy. Where the histology of a gastric ulcer is in doubt a frozen section biopsy should be taken before proceeding to definitive surgery (Fig. 2).

It is my pleasure to thank Dr. F. Avery Jones for access to his records, Dr. Richard Doll for personal communications, and Mr. A. G. Booker for the illustrations.

\section{BIBLIOGRAPHY}

ARMITAGE, G. (I953), Brit. med. F., March 14. AVERY JONES, F., et al. (I950), Ibid., January 28. AUCHINCLOSS, H. (1952), Ann. Surg., 135, No. I. DOLL, R. (1950), Brit. med. F., January 28. EMMETT, J. M., et al. (1953), Ann. Surg., 138, 320. FORGE, CLIFTON (1953), Ibid., 138, No. 3.

FORTY, FRANK (1946), Brit. med. F., May 25, p. 790.
GLEN, F., et al.

GRAHAM, R. R. (I946), 72, 802.

HENLEY, F. A. (1952), Brit. F. Surg., 40, No. 160.

HENLEY, F. A., (1953), Ann. R.C.S., 13, No. 3.

HESLOP, T. S. (1952), Brit. F. Surg., 40, No. 159.

ILLINGWORTH, C. F. N., et al. (I946), B.M.H., I, 787 .

IVY, GROSMAN and BACHRACH (I950), 'Peptic Ulcer.'

LASSEN, H. KRIEGER (I952), A.M.A. Arch. Surg., 65, No. 5.

LOWDON, A. G. R. (1952), Lancet, 1220.

MOORE, H. G., and HARKINS, H. (1952), 'The Bill roth I. Gastric Resection.'

NUBOER, J. F. (I95 I), Lancet, 952.

OLSON, H. B., and NORGORE, M. (1946), Ann. Surg., 124, 479

REA, C. E. (1952), Surg. St. Louis, 32, No. 4.

SPALOIZ, J. E. (1946), Lancet. i, 643.

TURNER, F. P. (1951), Surg. Gynae. Obst., 92, 28 I.

WALLENSTEN, S. (1952), Acta Chir. Scand., 104, Fase I.

WANGENSTREN, O. H. (1935), Minnesota Med., 18, 477.

WRIGHT, I.. T., and SCOTT, B. E. (I950), four. Pedia., 37, No. 6.

VISICK, A. HEDLEY (1946), Brit. med. F., p. 94 I.

\title{
¿ LARGE BOWEL OBSTRUCTION
}

\author{
By Guy Blackburn, M.Chir., F.R.C.S. \\ Surgeon, Guy's Hospital
}

The clinical picture of large gut obstruction is easily recognized in its classical and advanced form. Improvement in the results of treating it will come mainly from earlier diagnosis and acceptance of the fact that one of the triad of symptoms of colicky pain, distension and absolute constipation may often be sufficient to identify it. Vomiting, of course, is late and may merely signify secondary small intestine distension, because the ileo-caecal valve is no longer functioning efficiently. Alternatively, it may herald the onset of peritonitis from perforation or strangulation.

The most important maxim in dealing with potential cases of large gut obstruction is, firstly, to suspect it, when a patient has any one symptom of it, and secondly, to keep a close watch until the suspicion is dispelled or the diagnosis confirmed. Severe abdominal pain without diarrhoea is rarely of anything but surgical import and timely action in dealing with it may place a patient in surgical hands in good time to allow conservative measures to achieve relief, with subsequent elective surgery in optimum circumstances.

\section{Pathology}

Large gut obstruction occurs for the most part in elderly patients, the definition of elderly being in the seventh or eighth decades. This alone is the most important factor in prognosis, coupled with the fact that carcinoma of the colon is easily the commonest cause of the obstruction. This implies that occlusion is common and strangulation rare, the latter supervening in volvulus, band obstruction and rare examples of strangulated hernia. Umbilical hernia is the commonest of these, involving the transverse colon.

Local oedema and faecal impaction above an annular carcinoma of the pelvic colon convert a subacute into an acute obstruction. ${ }^{1}$ Distension above the growth may then result in perforation of the bowel, if the ileo-caecal valve is efficient enough to keep the loop ' closed' and not allow reflux of gas into the ileum. In these circumstances perforation of the pelvic colon or thinner and more distensible caecum (" pistol shot perforation') may dramatically complicate the picture, with dire results.

Carcinoma of the right half of the colon is more commonly papilliferous than annular and obstruction is correspondingly less frequent than on the left side ( $1: 7$ ) (Morgan). ${ }^{2}$ Rarely, in fact, is it the manner in which a patient with such a lesion presents, whereas IO-I 5 per cent. of patients with a ring carcinoma of the pelvic colon are first seen with large bowel obstruction. This may easily be confused with diverticulitis, though acute obstruc- 
tion with diverticulitis is rare. Perforation, pelvic abscess and vesico-colic fistula are commoner and these will draw attention to the condition before obstruction has occurred. In rare cases, even at operation, the nature of the obstructing lesion may not be identifiable with certainty, as diverticula may well be present as well as a neoplasm.

Mention must finally be made of faecal impaction as a cause of large bowel obstruction. Two cases have been seen by the writer, in the last six months, of previously unsuspected pyloric obstruction in septuagenerian patients presenting as faecal impaction. The presence of fluid levels in the colon in the plain X-ray of one of these had made the picture even more confusing.

\section{Clinical Considerations}

Enough has been said already to emphasize the importance of the patient's age in this condition. An alteration in the bowel habit is usual and a history of alternating constipation and diarrhoea said to be classical. Mythical might be more apposite, as it is too rare to warrant a mention. Rectal bleeding likewise is uncommon, unless it be from piles, as the growth that obstructs is scirrhus, and not ulcerated, and the one that bleeds is too soft and vascular to obstruct. Loss of weight, dehydration, disturbances of blood chemistry, anaemia and cachexia are all late symptoms of carcinoma of the pelvic colon, and the harbingers of impending obstruction are distension in the flanks, colic in the distribution of the transverse colon and a difficulty in passing flatus. One patient seen recently had bought herself a Higginson's syringe for the self-administration of enemata, as the widest range of aperients had failed to relieve her constipation and check the progressive abdominal distension, which she knew to be ' wrong in a woman of 62 .'

Physical signs supporting the diagnosis of large gut obstruction may not be easy of interpretation, if the patient be old and obese and the tone of the abdominal musculature poor. The rarity of hernia as a causative agent emphasizes this point, though hernial orifices should always be examined with care, as strangulated femoral hernia has often been mistaken for large gut obstruction. Obstructive symptoms in appendicitis in the aged may also give rise to difficulty. Rectal and pelvic examination, if possible bimanually, are vital, and instances where the proctoscope and sigmoidoscope may help towards solution of the problem of everyday experience.

Abdominal palpation will not as a rule reveal a mass, and local tenderness, muscular rigidity, fever and signs of local or general peritoneal irritation are associated with strangulation rather than simple occlusion. Visible peristalsis must also be clearly interpreted, as it is almost invariably the contraction of the small intestine that is being $\vec{z}$ observed. Very thin patients and those with $a \stackrel{\mathbb{Q}}{\varrho}$ strangulated umbilical hernia provide the ex-.. ceptions.

\section{Radiology}

Plain films of the abdomen, preferably in the erect position, are invaluable in locating the site $\frac{D}{\vec{D}}$ of obstruction. Where the patient is unable to $\unrhd$ stand in the X-ray department, a film taken in sitting up or even lying on the side in bed may $\overrightarrow{0}$ be sufficient to provide the necessary information. Conservative negotiation of the obstructive phase without operation may allow a confirmatory barium enema. Even the administration of such an enema in the presence of obstruction carries very little risk, but that does not recommend it. $\stackrel{\omega}{\omega}$ The converse is, of course, true of barium by $\dot{\omega}$ mouth: and a barium meal or follow-through, in or a suspected case of carcinoma of the pelvic colon, i may well be the means of precipitating an obstruc- $\vec{\circ}$ tion. Should this disaster befall, the liberal $\frac{\text { ? }}{4}$ administration of paraffin by the mouth and olive $\rightarrow$ oil in enemata per rectum may sometimes avert untimely surgical intervention.

The level at which the gas shadows stop in the $\overrightarrow{0}$ colon, rather than the presence of fluid levels, the most important information conveyed by. plain X-ray, but the test is far from infallible Fluid levels may be the result of the "two enema test' before X-ray or sometimes disappear, when the bowel is washed out. They are far less instructive than in the small intestine.

A coin on the umbilicus may materially assist in planning the incision for a transverse colostomy and a Ryle's tube in the stomach may be helpful in reducing the size of an obscuring gastric shadow.

\section{Diagnosis}

Laboratory investigations are, as a rule, relatively unimportant in the diagnosis of obstruction of the large intestine, but instances of haemoconcentration from fluid loss, nitrogen retention and hypochloraemia from vomiting will be observed in cases brought to hospital late. Physical examination with radiological help will $N$ really be the means of deciding what course of $N$ action should be taken. In this connection, the time factor is of paramount importance, particularly where strangulation is suspect, as in volvulus. In these circumstances temporization with venoclysis and intestinal suction may give a false sense of security and it is safer to proceed with laparotomy, where unusual features complicate the clinical picture. Such was the case in a man of 64 years of age, admitted to hospital a few hours 
after consulting his doctor on account of distension of the lower abdomen and an inability to pass water. The presence of a palpable tender swelling in the left iliac fossa, without any prostatic enlargement per rectum, confirmed that the history of recent bowel irregularity was probably due to diverticulitis. Emptying the bladder by catheter failed to relieve the distension much and laparotomy revealed a perforated diverticulitis, for which transverse colostomy and drainage was carried out. In spite of this, death occurred in a few hours, although the whole history was less than 24 hours long.

\section{Treatment}

Simple occlusion often responds to suction and intravenous fluids, but hospitalization is the prime requirement. Administration of more than one enema is often unwise, as it wearies the patient and achieves nothing. Olive oil and gravity may be far better. If the bowel below the obstructing lesion shows impaction or, if impaction by itself be the cause of the obstruction, manual removal under anaesthesia may be the only solution. In octogenarian and infirm psychotic patients this operation is often required in mental hospitals. Volvulus is also said to be commoner in these institutions than elsewhere. Correction by a tube per rectum introduced through a sigmoidoscope is not without risk and perforation has been described. In volvulus due to long loops of redundant colon in younger patients, spontaneous reduction by a trick movement and the adoption of some curious position by the patient may at times be corrective. Loud borborygmi and the passage of a great deal of flatus signify that success has been achieved.

After correction of an impending large bowel obstruction by non-operative measures there is time for oral administration of phthalylsulphathiazole or succinylsulphathiazole to prepare the colon for elective resection. Oral streptomycin is a useful adjunct and some authorities have advocated the broad-spectrum antibiotics. None of these has any place, however, in the presence of acute obstruction.

Operative methods have changed since the advent of antibiotics, and intraperitoneal resection after preliminary colostomy is now safe. It has thus taken the place of the Paul-Mikulicz procedure, which was often an unsatisfactory cancer operation in respect of the area of tissue excised. It is not, however, obsolete in some cases of volvulus and, rarely, obstruction by growth in old and enfeebled patients, where coincident cardiovascular and other debilitating conditions make lengthy procedures risky.

\section{Colostomy}

Discussion has raged over the years about the value of caecostomy in acute large bowel obstruction. 'Blind' caecostomy certainly has few adherents left and the author is not among them. ${ }^{3}$ On the other hand, a caecostomy with adequate exteriorization of a part of the caecum may at times be safer than ileo-transverse colon anastomosis as a first step in removing a carcinoma of the right side of the colon, causing obstruction.

Transverse colostomy has correspondingly gained favour, as exteriorization of a loop with a glass rod produces good defunctioning of the distal loop, which can then be prepared for resection. Where diverticulitis has been the indication for operation, with obstruction as a complication, the colostomy is likely to have to remain for a longer time. Resection of areas of colon locally affected with diverticulitis is more commonly practised than before the days of antibiotics and the interval between colostomy and resection here should be far longer than in carcinoma.

Inguinal colostomy is, generally speaking, open to the objection that it has to be established too near the growth for convenience, either of satisfactory faecal diversion or subsequent radical surgery, unless the colostomy is resected with the growth.

\section{Resection}

Advocates of primary resection in carcinoma of the colon with obstruction, point to the overall mortality of other methods of treatment and stage operations. This ranges round 20-25 per cent. and the fact that only 17 of a recent series of 32 cases, admitted to Guy's Hospital ${ }^{4}$ in obstruction due to colon cancer, left hospital with the primary growth resected is a simple illustration of the need for improvement in this condition. The cause of the mortality and morbidity, however, lies in the fact of late diagnosis and not in the nature of operative procedures, for none of the deaths in the 32 cases occurred immediately after operation.

Primary resection on the right side has more in its favour than on the left and Muir's ${ }^{5}$ ileal suction drainage after right hemicolectomy is a valuable safety measure, equivalent to an ileostomy drain. Leakage is said to be very unusual, when the tube is removed 7-Io days later.

End-to-end anastomosis is efficient and time saving after right hemicolectomy and disparity in the lumen of the colon and ileum can be corrected by oblique section of the latter. Blood supply is no real problem on this side and the marginal artery may well be sufficient, even if the middle colic artery is sectioned. The step should be taken early in the operation, to establish the point with certainty. Transverse colostomy as a primary 
measure for decompression of the colon involves resection of the colostomy at a later date, when closure is required. This, however, is easy, as is closure of a caecostomy, where partial exteriorization has been carried out. Spontaneous or extraperitoneal closure of either, where exteriorization in the first instance was incomplete, is often less secure.

The place of resection of the pelvic colon in the presence of obstruction has been championed by dexterous enthusiasts, who claim excellent results. It must be remembered, however, that many emergency operations are performed by registrars and young surgeons, in circumstances far less easy for the patient than those obtaining for prepared elective resection in the light of day. Safety must, therefore, lie in the advocacy of preliminary transverse colostomy, where any element of obstruction is present. Full exploration of the abdomen is neither necessary nor advisable in all cases at the first intervention, as the presence of liver metastases is no bar to resection of the colon growth causing obstruction. The liver can, therefore, be fully examined at the subsequent resection, when other evidence of spread can be sought, the presence or absence of second growths determined and fixation to adjacent structures accurately assessed.

\section{Non-Malignant Affections of the Large Bowel Causing Obstruction}

Volvulus, band obstruction, intussusception, strangulated umbilical hernia and diverticulitis are the commonest of these and the last is dealt with in a manner very similar to carcinoma. Transverse colostomy is advisable for the management of most of its complications. Where the two conditions coexist, diagnosis may sometimes be missed, but obstruction by itself demands a course of action applicable to either. Colloid carcinoma occurring in ulcerative colitis may likewise give rise to a confusing clinical picture.

Regional colitis, in the absence of regional ileitis, is rare and should be treated conservatively, if recognized in an obstructive phase. If found as an accidental discovery at laparotomy, it is probably wise to close the abdomen and defer resection until suitable preparation has been carried out. One patient, who was found to have this condition at laparotomy, went a further four months before coming to resection for obstructive symptoms. The histology of the bowel lesion closely resembled that of typical Crohn's disease.

\section{Anaesthesia}

Inhalation pneumonia was at one time a common sequel of operations for acute large gut obstruction. Some surgeons, therefore, advocated local anaesthesia, and spinal anaesthesia had a vogue. Neither would seem necessary now that cuffed endotracheal tubes, bronchoscopic suction and relaxants, without bronchial irritants, are so inherent a part of the anaesthetist's armamentarium. Patients with large gut obstruction, however, still provide difficult anaesthetic problems, closure of the abdomen even after a surgical decompression being at times very difficult. A Ryle's tube is a sine qua non of the stage of induction, or an oesophageal tube, passed under anaesthesia, if the patient cannot tolerate the first. It is also important that suction should be continued until post-operative deflation of the bowel has occurred. The Miller-Abbot tube is generally unsatisfactory, as it is difficult to pass and will not negotiate the ileo-caecal valve, but the Ryle's tube is a good alternative. A rectal tube may also help in deflating the bowel below the site of obstruction. In certain cases of volvulus it will with the sigmoidoscope, render surgery un necessary.

\section{Results}

The results of treating acute large bowel obstruction by colostomy, followed by resection in I4 days on the left side and ileo-transverse colostomy with resection at the same interval on the right side, still leave room for improvement. This will come mainly from earlier diagnosis, laparotomy in doubtful lesions of the colon (e.g. the splenic. flexure), where sigmoidoscopy and radiology are inconclusive, and strict attention to the details of conservative decompression, where strangulation can be ruled out. Abandoning the Paul-Mikulicz. operation in favour of colostomy and subsequent wider resection should likewise improve ultimate prognosis in many of these patients.

\section{REFERENCES}

I. SMITH, RODNEY (1954), 'Progress in Clinical Surgery, Churchill.

2. MORGAN, C. N. (1952), Ann. R. Coll. Surg. Eng., 10, 305. 3. BLACKBURN, G. (1952), Trans. Med. Soc. London, 69, 38.

4. LANE-ROBERTS, P. A., Personal communication.

5. MUIR, E. G. (1947), Proc. Roy. Soc. Med., 40, 831 . 